\title{
Administration of Bifidobacterium bifidum BGN4 and Bifidobacterium Iongum BORI Improves Cognitive and Memory Function in the Mouse Model of Alzheimer's Disease
}

\author{
Hongwon Kim ${ }^{1,2}$, Sumin Kim², Sang-jun Park ${ }^{3}$, Gwoncheol Park ${ }^{4}$, Hakdong Shin ${ }^{4}$, \\ Myeong Soo Park ${ }^{3 *}$ and Jongpil Kim ${ }^{1,2 *}$
}

\begin{abstract}
'Department of Biomedical Engineering, Laboratory of Stem Cells and Cell Reprogramming, Dongguk University, Seoul, South Korea, ${ }^{2}$ Department of Chemistry, Dongguk University, Seoul, South Korea, ${ }^{3}$ Research Center, BIFIDO Co., Ltd, Hongcheon, South Korea, ${ }^{4}$ Department of Food Science and Biotechnology, Sejong University, Seoul, South Korea
\end{abstract}

OPEN ACCESS

Edited by:

George Tetz,

Human Microbiology Institute,

United States

Reviewed by:

Yashi Mi,

University of Arizona,

United States

Xavier Xifró,

University of Girona,

Spain

${ }^{*}$ Correspondence: Jongpil Kim

jk2316@gmail.com; jpkim153@dongguk.edu

Myeong Soo Park

bifidopark@bifido.com

Received: 13 May 2021

Accepted: 15 July 2021

Published: 06 August 2021

Citation:

Kim H, Kim S, Park S-j, Park G,

Shin H, Park MS and Kim J

(2021) Administration of

Bifidobacterium bifidum BGN4 and

Bifidobacterium longum BORI

Improves Cognitive and Memory

Function in the Mouse Model of

Alzheimer's Disease.

Front. Aging Neurosci. 13:709091.

doi: 10.3389/fnagi.2021.709091
Recent evidence indicates that gut microbiota could interact with the central nervous system and affect brain function, including cognition and memory. In this study, we investigated whether Bifidobacterium bifidum BGN4 (B. bifidum BGN4) and Bifidobacterium longum $\mathrm{BORI}(\mathrm{B}$. longum $\mathrm{BORI})$ alleviated the pathological features in a mouse model of Alzheimer's disease (AD). Administration of B. bifidum BGN4 and $B$. longum BORI effectively suppressed amyloidosis and apoptotic processes and improved synaptic plasticity by ameliorating the neuroinflammatory response and BDNF expression. Moreover, behavioral tests indicated that B. bifidum BGN4 and B. longum $\mathrm{BORI}$ attenuated the cognitive and memory disability of $A D$ mice. Taken together, the present study highlights the therapeutic potential of B. bifidum BGN4 and B. longum $\mathrm{BORI}$ for suppressing the pathological features of $\mathrm{AD}$.

Keywords: Alzheimer's disease, probiotics, gut microbiota, Bifidobacterium, cognitive and memory impairment

\section{INTRODUCTION}

Alzheimer's disease (AD) is a progressive neurodegenerative disorder that causes cognitive decline and memory loss. Its major pathological hallmarks are an accumulation of amyloid peptides, which are products of amyloid precursor protein (APP), and intracellular neurofibrillary tangles of hyperphosphorylated tau protein (Jucker and Walker, 2011). Mutations in APP, presenilin, and tau genes cause the development of relevant molecular pathologies inside neurons, leading to neuroinflammation and altered synaptic plasticity, and eventually resulting in neuronal death (Goate et al., 1991; Wolfe et al., 1999). Previous studies have shown that alternative therapies, including acupuncture, meditation, and medical foods, can treat AD (Thaipisuttikul and Galvin, 2012; Jia et al., 2017; Innes et al., 2018). Additionally, dietary supplements, such as unsaturated fatty acids, and vegetables, legumes, fruits, and omega prevent AD by reducing LDL cholesterol levels, have antioxidant and anti-inflammatory effects, and attenuate cognitive decline (Hu et al., 2013; El-Sayyad, 2015; Mendiola-Precoma et al., 2016). 
Recently, studies have implicated gut microbiota in several neurological disorders, such as AD (Vogt et al., 2017), autism spectrum disorder (Sgritta et al., 2019), and Parkinson's disease (Lee et al., 2019). Based on the concept of the microbiota-gut-axis, the relationship between the CNS and gut microbiota contributes a key role in disease risk, and the activity and progression of neurological disorders. For example, the gut microbiota, including Ruminococcaceae, Rikenellaceae, Clostridiaceae, and Enterobacteriaceae, acts as key regulators of neuroinflammation in aged mice (Conley et al., 2016; Matt et al., 2018). Moreover, an increase in the abundance of the pro-inflammatory gut microbiota taxon, Escherichia/Shigella, accelerates brain amyloidosis in cognitively impaired elderly individuals (Cattaneo et al., 2017). Moreover, many studies showed that the altered gut microbiota secretes immunogenic compounds such as amyloid, lipopolysaccharides (LPS), and other microbial exudates which mediate the effects of microbiota in several neurological disorders. For example, LPS can be translocated from the gut to the brain, exacerbating amyloid deposition in the AD model (Zhan et al., 2016). Sgritta et al. (2019) also reported that alteration of L. reuteri in gut microbiota modulates the expression of oxytocin via the vagus nerve in mouse models of autism spectrum disorder (Sgritta et al., 2019). Additionally, truncal vagotormy in mice prevented the spread of $\alpha$-synucleinopathy from the gut to the brain (Kim et al., 2019), indicating that these immunogenic compounds mediate afferent and efferent pathways such as the vagus nerve or the hypothalamic-pituitary adrenal pathway (Grenham et al., 2011; Sgritta et al., 2019). Taken together, these results suggest that alterations in gut microbiota composition by probiotics may provide a novel therapeutic approach or ameliorating strategy for $\mathrm{AD}$. Consistent with these results, recent studies have shown that the treatment of Bifidobacterium breve strain A1 alters gut microbiota and ameliorates cognitive dysfunction in an $A \beta$ induced AD mouse model (Kobayashi et al., 2017). In addition, the administration of xylooligosaccharides attenuates surgeryinduced cognitive dysfunction in APP/PS1 AD mice by altering intestinal microbiota (Han et al., 2020). Moreover, in one of our previous our study, probiotic supplementation containing B. bifidum BGN4 and B. longum BORI was shown to improve mental flexibility and alleviate stress in healthy older adults (Kim et al., 2021). Therefore, from this perspective, we asked whether probiotics containing B. bifidum BGN4 and B. longum BORI can alleviate $\mathrm{AD}$ pathologies, such as cognitive dysfunction and memory loss.

In this study, in order to examine the therapeutic effects of $B$. bifidum BGN4 and B. longum BORI administration for $\mathrm{AD}$, probiotic supplementation containing $B$. bifidum BGN4 and B. longum BORI was treated by oral administration in a mouse model of $\mathrm{AD}$. We first determined the effects on the suppressed amyloidosis and apoptotic process in the hippocampus of AD mice. We subsequently examined behavioral changes using the Y-maze, fear conditioning, and Morris water maze tests in probiotics treated $\mathrm{AD}$ mice. Importantly, we found that treatment with B. bifidum BGN4 and B. longum BORI significantly improved the cognitive and memory ability of $5 \times \mathrm{xAD}$ mice, indicating that the administration of probiotic
B. bifidum BGN4 and B. longum BORI in gut microbiota can effectively ameliorate the pathological features of AD.

\section{MATERIALS AND METHODS}

\section{Animals and Treatment Protocol}

Five familial AD mutations (APP K670N/M671L, V717I, I716V, and PS1 harboring two FAD mutations, M146L and L286V)expressing $5 x F A D$ transgenic mice were obtained from The Jackson Laboratory (Bar Harbor, ME). Age- and sex-matched C57BI/6 and 5xFAD mice were used for all experiments and randomly assigned to each group. The mice were assigned into four groups: Control-BGN4/BORI group $(n=10)$, Control+BGN4/BORI group $(n=10), 5 x F A D-B G N 4 / B O R I$ group $(n=10)$, and $5 x F A D+B G N 4 / B O R I$ group $(n=10)$. For probiotic treatment, freeze dried $B$. bifidum BGN4 and $B$. longum BORI powder (BIFIDO, Gangwon, Korea) were orally administrated to 3 -month-old mice daily via oral gavage $\left(1 \times 10^{9}\right.$ CFU in $0.2 \mathrm{ml}$ sterile water) for 30 days. All experiments were performed in accordance with the institutional guidelines for animal use and received ethical approval from the Institutional Animal Care and Use Committee at Dongguk University.

\section{Genomic DNA Extraction}

To collect murine fecal samples, each group was placed in a separate sterile cage. The mice were assigned into four groups: Control-BGN4/BORI group, Control+BGN4/BORI group, 5xFAD-BGN4/BORI group, and 5xFAD+BGN4/BORI group. Fecal pellets (for $n=3$ per group) were collected directly from the anal orifices once a week for 5 weeks and stored at $-80^{\circ} \mathrm{C}$ for analyses. Total bacterial DNA was isolated from the stool by using ZymoBIOMICS ${ }^{\mathrm{TM}}$ DNA Miniprep Kit (D4304, Zymo Research Corporation, Irvine, USA). The total DNA was isolated and purified following the manufacturer's protocol. After extraction, the DNA purity and concentration were measured with a spectrophotometer, Nano-Drop ND-2000 (Thermo Scientific, Waltham, MA) and Qubit 3.0 fluorometer (Thermo Scientific). Samples were stored at $-20^{\circ} \mathrm{C}$ until further analysis.

\section{Amplification of 16S rRNA Gene and Sequencing}

16S rRNA gene amplification and index PCR were conducted following the Illumina 16S Metagenomic Sequencing Library preparation guide (Illumina, San Diego, CA, USA). The $16 \mathrm{~S}$ sequence was amplified using forward primer and reverse primer. PCR was initially performed using the primer set, MiSeq 341F (5'-TCGTCGGCAGCGTCAG ATGTGTATAA GAGACAGCCTACGGGNGGCWGCAG-3') and MiSeq 805R (5'-GTCTCGTGGGCTCGGAGATGTGTATAAGAGACAG GACTACHVGGGTATCTAATCC- $3^{\prime}$ ) using $2 \times$ Kapa $\mathrm{HiFi}$ Hot start Ready Mix DNA polymerase (Kapa Biosystems, Wilmington, MA, USA). PCR products were purified using the Agencourt AMPure XP kit (Beckman Coulter, Brea, CA, USA). Amplification was performed at $95^{\circ} \mathrm{C}(3 \mathrm{~min})$ with 25 cycles of $95^{\circ} \mathrm{C}(30 \mathrm{~s}), 55^{\circ} \mathrm{C}(30 \mathrm{~s}), 72^{\circ} \mathrm{C}(30 \mathrm{~s})$, and a final extension of $72^{\circ} \mathrm{C}(5 \mathrm{~min})$. Quantification and size estimation of the library 
was carried out on the QIAxcel Advanced using QIAxcel DNA High Resolution Kit (QIAGEN, Hilden, Germany). Sequencing was performed using the Illumina MiSeq System $(2 \times 250 \mathrm{bp}$ paired-end reads; Illumina, USA).

\section{Bioinformatic Analysis of Sequencing Data}

Microbial sequences were processed using QIIME2 (version 2020.6). Raw sequence reads were demultiplexed using the q2-demux plugin, followed by denoising to remove the sequences with low-quality score using DADA2. All amplicon sequence variants (ASVs) were aligned using the MAFFT and were used to create a rooted phylogenetic tree for phylogenetic diversity analysis with FastTree 2 (Price et al., 2010). ASVs were assigned based on pre-built branches from the 99\% SILVA 132 database with a naïve Bayes taxonomy classifier developed for the sklearn classifier (Bokulich et al., 2018). All samples were rarefied to a maximum depth (19,431 reads) that could retain all samples. Faith's phylogenetic diversity and observed features were calculated to measure microbial diversity. Unweighted and weighted UniFrac distance metrics were used to compare the microbial community structure (Lozupone and Knight, 2005; Lozupone et al., 2007). The non-parametric Kruskal-Wallis test was used to determine significant differences in microbial diversity. To evaluate the difference in community structure, PERMANOVA (with 999 random permutations) was used. The linear discriminant analysis effect size (LEfSe) method and LDA effect size (cut-off $\geq 3$ ) was used to detect significant differences in comparison to the bacteria abundance (Segata et al., 2011). Correlations between gut microbiota and parameters were calculated by using the Spearman's rank correlation coefficient in $\mathrm{R}$ package.

\section{Accession Number}

The accession number for the gut microbiome data reported in this article is PRJNA731317.

\section{Behavioral Tests}

The Y-maze test was performed 30 days after probiotic administration to assess short-term memory. Spontaneous alternation and the number of alternations were tested using white polyvinyl plastic with three open arms (300 $\mathrm{mm}$ deep, $150 \mathrm{~mm}$ high, $50 \mathrm{~mm}$ wide,) at angles of $120^{\circ}$ from each other. Mice were placed at the end of one arm and recorded during the 10 -min test period. The maze was washed with $70 \%$ ethanol after each trial. Data were acquired using the Y-maze software. The sequence of arm entry and the total number of entries was counted by the software to calculate the alternation ratio.

A contextual fear conditioning test was performed using standard chamber with shock floors $(20 \times 20 \mathrm{~cm})$ and conducted over 3 days. On the 1 st day, each mouse was placed in a fear conditioning chamber for $5 \mathrm{~min}$ and exposed to white background noise. On the 2nd day of the conditioning session, mice received a $1-\mathrm{s} 1.7 \mathrm{~mA}$ electric foot shock at $3 \mathrm{~min}$. The training was repeated twice. Twenty-four hours later, mice were exposed to the chamber for $5 \mathrm{~min}$ without an electric foot shock. The freezing behavior of mice was videotaped, and the freezing and exploring in the fear conditioning chamber was calculated using specialized behavioral tracking software (EthoVision).
The Morris water maze was performed to test the acquisition of spatial memory and long-term spatial memory. This test was performed in a circular pool (diameter: $90 \mathrm{~cm}$, height: $45 \mathrm{~cm}$ ) filled with water. An escape platform was placed below the water surface. The water maze test was conducted over 5 days with three trials per day. Each trial was recorded over a $60 \mathrm{~s}$ test period. Twenty-four hours after the last training trial, mice were exposed to a $60 \mathrm{~s}$ probe test, in which the platform had been removed from the pool. Performance in the probe trial of the water maze was videotaped. The time spent in each quadrant was analyzed using specialized behavioral tracking software (EthoVision).

\section{Western Blot Analysis}

Mouse brains were lysed in RIPA buffer containing 1\% NP-40, $0.5 \% \mathrm{DOC}, 0.1 \% \mathrm{SDS}$, and $150 \mathrm{mmol} / \mathrm{L} \mathrm{NaCl}$ in $50 \mathrm{mmol} / \mathrm{L}$ Tris $(\mathrm{pH}$ 8.0) supplemented with $1 \times$ proteinase inhibitor mixture (GenDepot). After adding $5 \times$ SDS loading buffer and boiling for $5 \mathrm{~min}$, the samples were centrifuged for $10 \mathrm{~min}$ at $12,000 \mathrm{~g}$. The supernatants were electrophoresed on $7.5 \%$ sodium dodecyl sulfate-polyacrylamide gel and transferred to $0.2 \mu \mathrm{m}$ nitrocellulose membranes. The membrane was then probed with the following antibodies: Homer1 (1:1,000, Millipore, ABN37), PSD95 (1:1,000, Cell Signaling, 2507S), BDNF (1:1,000, Invitrogen, OSB00017W), APP c-terminal (1:500, Sigma, A8717), and $\beta$-actin (1:1,000, Abfrontier, LFPA0207).

\section{Immunofluorescence Staining Analysis}

Brain samples of control and $5 \mathrm{xFAD}$ mice were sliced to $40 \mu \mathrm{m}$ with a microtome. Brain sections were washed with $1 \times$ phosphate-buffered saline after being fixed in $4 \%$ paraformaldehyde in phosphate-buffered saline. Brain sections were immunostained according to standard protocols using the following primary antibodies: ChAT (Millipore, AB144P), NeuN (Millipore, MAB377), MAP2 (Invitrogen, 13-1,500), BDNF (Invitrogen, OSB00017W), $\beta$-amyloid 1-42 (Biolegend, 805501), Cleaved-caspase3 (Cell Signaling, 9661S) and appropriate fluorescent secondary antibodies (Invitrogen). Next, brain sections were mounted in Fluoromount-G mounting medium. Representative images were then captured using a confocal laser-scanning microscope (ZEISS, LSM800). The number of ChAT-expressing neurons and NeuN-expressing cells was assessed in the hippocampal CA1, CA2, and CA3 region using the automatic cell counter plugin in ImageJ. An investigator blinded to the experimental conditions obtained bilateral counts of ChAT- and NeuN-ir from anatomically matched images to produce an average score.

\section{Quantitative RT-PCR Analysis}

Total RNA for all conditions was extracted from the hippocampus and purified using an RNeasy Kit (QIAGEN) according to the manufacturer's protocols. The AccuPower RT-PCR PreMix (Bioneer) was used to synthesize cDNA from isolated RNA. Quantitative RT-PCR analysis was performed using Platinum SYBR green qPCR SuperMix (Invitrogen) in a Rotor-Gene Q real-time PCR cycler (QIAGEN) with following conditions: $95^{\circ} \mathrm{C}$ for $15 \mathrm{~min}$ followed by 40 cycles of $95^{\circ} \mathrm{C}$ for $10 \mathrm{~s}, 55^{\circ} \mathrm{C}$ for $15 \mathrm{~s}, 72^{\circ} \mathrm{C}$ for $20 \mathrm{~s}$; melting curve from $72^{\circ} \mathrm{C}$ 
to $95^{\circ} \mathrm{C}$ every $0.2^{\circ} \mathrm{C}$ for $1 \mathrm{~s}$ per step. Target gene expression was normalized against the expression of a housekeeping gene, GAPDH, in control and 5xFAD mice administrated with sterile water or B. bifidum BGN4 and B. longum BORI. The following PCR primers were used: $I L-17$ Forward: $5^{\prime}$-CCAGGGAGAG CTTCATCTGT-3' Reverse: 5'-AGGAAGTCCTTGGCCTCA GT-3', IL- $1 \beta$ Forward: 5'-GGATGAGGACATGAGCAA CCT-3' Reverse: $5^{\prime}$-AGCTCATATGGGTCCGACAG-3', IL-6 Forward: 5'-CCGGAGAG GAGACTTCACAG-3' Reverse: 5' -CAGA ATTGCCATTGCACAAC-3', IL-10 Forward: 5'-TGCTGCCT GCTCTTACTGAC-3' Reverse: 5'-TGGCAACCCAAGTAAC C CTT-3', NF- $k B$ Forward: 5'-AGGCTCCTGTGCGTGTCT CC-3' Reverse: 5'-AGGTCCACTGCG AGGTGAAGG-3', COX2 Forward: 5' -CTACAAGACGCCACATCCCC-3' Reverse: 5'-AT GCGTAGAGAGGGGAGAGC-3'.

\section{TNF- $\alpha$ and IL-12 Quantification}

ELISA was performed using a cytokine ELISA combo kit (KOMA Biotech, K033KIT-02) to detect TNF- $\alpha$ and IL-12 concentrations. Whole blood samples were collected from sterile water or probiotic-treated mouse hearts. Serum samples were separated by centrifugation at $1,000 \mathrm{~g}$ for $10 \mathrm{~min}$ in a refrigerated centrifuge. The collected samples were assessed via TNF- $\alpha$ and IL-12 ELISA kits, according to the manufacturer's instructions.

\section{Statistical Analysis}

Data are expressed as the mean \pm SEM of three independent experiments. One-way analysis of variance (ANOVA) followed by Tukey-Kramer multiple comparisons test was performed with GraphPad Prism (La Kolla, California, USA). $N$ values represent the number of independent experiments, number of individual experiments, or mice. All statistical details of experiments can be found in the figure legends.

\section{RESULTS}

\section{B. bifidum BGN4 and B. longum BORI Reduced Hippocampal Neuronal Death in 5xFAD Mice}

To assess the effect of B. bifidum BGN4 and B. longum BORI in $\mathrm{AD}$, we first examined the number of hippocampal neuronal cells in the CA3 region of 3 months old probiotic-treated $5 x F A D$ mice. Thirty days after BGN4/BORI treatment (once a day, $1 \times 10^{9} \mathrm{CFU}$ in $0.2 \mathrm{ml}$ sterile water), we observed significantly increased NeuN positive cells in the CA3 region of probiotic-treated $\mathrm{AD}$ mice hippocampus (Figures 1A,B). Since choline acetyltransferase (ChAT), the enzyme that synthesizes acetylcholine, is expressed during hippocampusbased learning and memory processes (Hawley et al., 2015), we next examined the number of ChAT+ neurons in the AD hippocampus. Consistent with previous results, we observed a significant increase in $\mathrm{ChAT}+/ \mathrm{NeuN}+$ cells in the CA3 region of probiotic-treated 5xFAD mice hippocampus (Figures 1A,C). Moreover, we confirmed a significant increase in the number of $\mathrm{ChAT}+/ \mathrm{NeuN}+$ cells in the CA1 region of probiotic-treated $5 x F A D$ mice (Figures 1D-F). However, we observed that the number of $\mathrm{NeuN}+$ and $\mathrm{ChAT}+/ \mathrm{NeuN}+$ cells was not changed in the CA2 region between groups (Figures 1G-I). These data suggest that $B$. bifidum BGN4 and B. longum BORI attenuated hippocampal neuronal death in $\mathrm{CA} 3$ and $\mathrm{CA} 1$ regions of $\mathrm{AD}$ mice.

\section{B. bifidum BGN4 and B. longum BORI Restored BDNF and Synaptic Scaffolding Proteins in the Hippocampus of 5xFAD Mice}

To evaluate the effect of B. bifidum BGN4 and B. longum BORI on functional synaptic plasticity, we next examined the expression level of BDNF, which regulates an important role in hippocampal synaptic plasticity and cognition (Vaynman et al., 2004). We found a significant increase in the number of Map2+/BDNF+ neurons in the hippocampus of $5 x F A D$ mice treated with $B$. bifidum BGN4 and B. longum BORI when compared with control-treated $5 \mathrm{xFAD}$ mice (Figures 2A,B). Semiquantitative Western blotting confirmed the increased protein expression of BDNF in the hippocampus of probiotictreated 5xFAD mice (Figures 2C,D). Further, the expression of synaptic scaffolding proteins, including PSD95 and Homer1, was significantly restored in probiotic-treated $5 \mathrm{xFAD}$ mice (Figures 2C,E), demonstrating that B. bifidum BGN4 and $B$. longum BORI improved the molecular composition of postsynaptic machinery in the mouse model of AD.

\section{B. bifidum BGN4 and B. longum BORI Ameliorated the AD Phenotypes of 5xFAD Mice}

Next, we examined whether treatment of B. bifidum BGN4 and $B$. longum BORI can ameliorate the $\mathrm{AD}$-associated phenotypes in the hippocampus of $5 \mathrm{xFAD}$ mice. Initially, we found reduced amyloid- $\beta 42$ positive cells in the hippocampus of $\mathrm{AD}$ mice at 4 weeks after the oral administration of probiotics (Figures 2F,G). Additionally, we observed a decrease in cleaved caspase- 3 positive cells, which are expressed in the apoptotic process, in probiotic-treated $5 x$ FAD mice (Figures 2 F,H). The production of APP-CTFs, C99 and C83, which are cleaved by $\beta$ - and $\alpha$-secretase contributes to the accumulation of amyloid aggregates (Checler, 1995; Lauritzen et al., 2012). Detection of C99 and C83 using the APP c-terminal antibody revealed a decrease in $\mathrm{C} 99$ protein expression in the hippocampus of probiotic-treated $5 \mathrm{xFAD}$ mice (Figures $2 \mathrm{I}-\mathbf{K}$ ). These results indicated that $\mathrm{AD}$ pathology was attenuated by $B$. bifidum BGN4 and B. longum BORI administration.

Next, we investigated the effect of $B$. bifidum BGN4 and $B$. longum BORI on brain inflammatory responses in $\mathrm{AD}$ mice. Previous studies have shown that the neuroinflammatory cascade aggravates $\mathrm{AD}$ pathogenesis, reduces neuroprotective factors, enhances synaptic dysfunction, and causes neuronal damage, leading to neurodegeneration (Kauwe et al., 2014; Chen et al., 2015). Thus, we determined the expression of inflammatory related genes and cytokines in the probiotic-treated $5 \mathrm{xFAD}$ mice. We first observed a statistically marginal increase in the expression of these genes from $5 x F A D$ mice compared to control mice $(n=5)$. Interestingly, B. bifidum BGN4 and B. longum BORI 

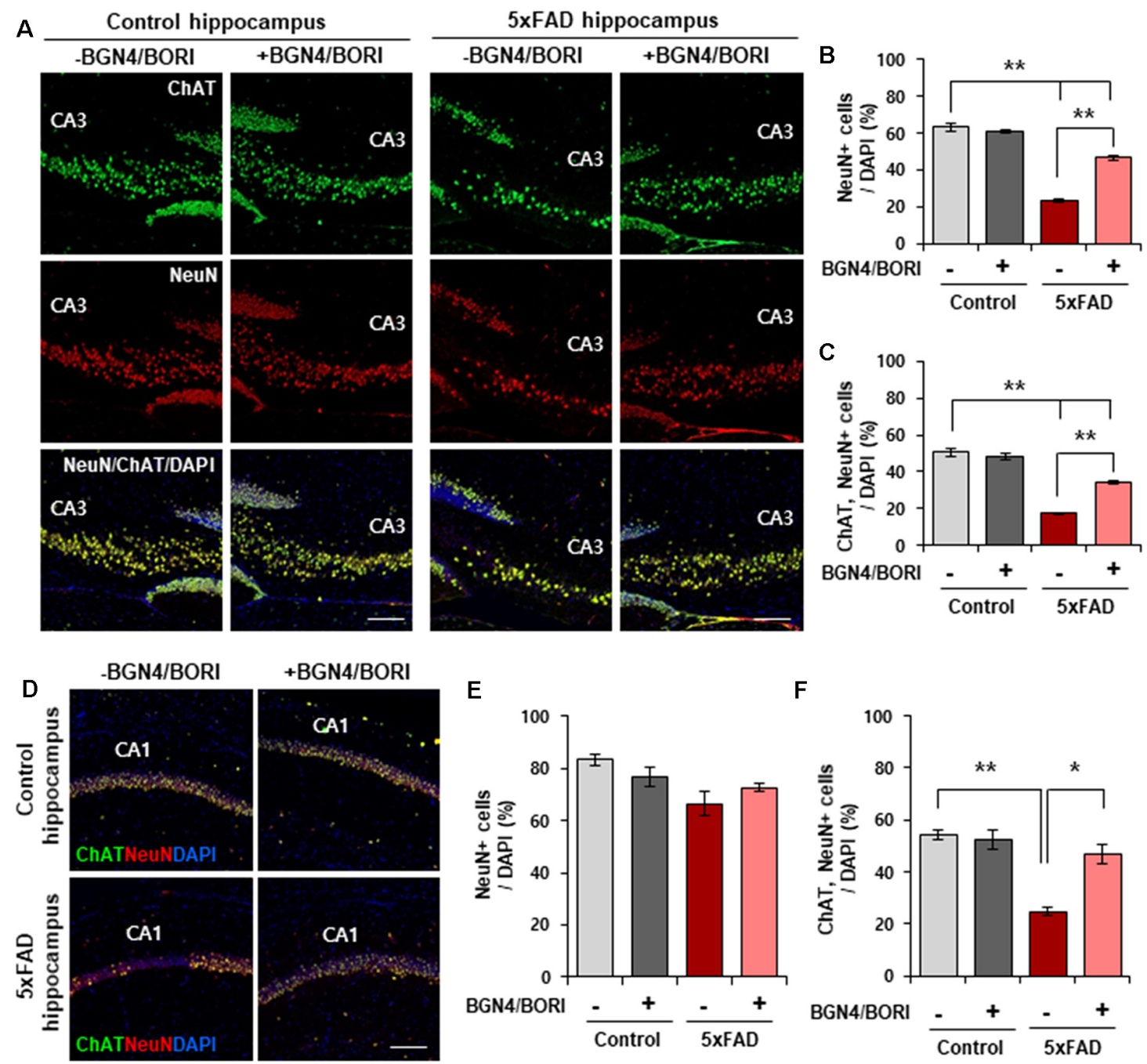

BGN4/BORI $\frac{-+}{\text { Control }} \frac{-}{5 \times F A D}$
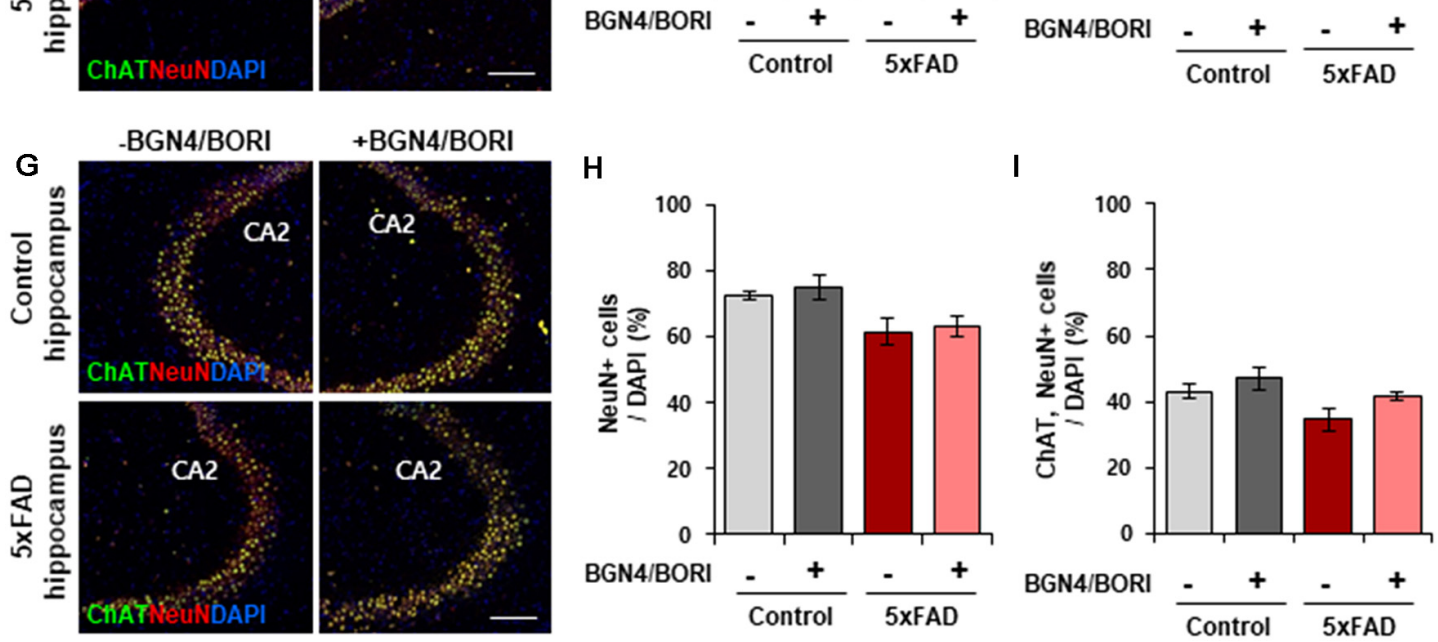

FIGURE 1 | B. bifidum BGN4 and B. longum BORI reduced hippocampal neuronal death in 5xFAD Mice. (A) Immunofluorescence for ChAT and NeuN positive cells in the CA3 hippocampal subfields of control (left) or 5xFAD mice (right) treated with B. bifidum BGN4 and B. longum BORI (BGN4/BORI). Scale bar = 50 $\mu$ m. (B,C) Quantifications of the $\mathrm{NeuN}+\mathbf{( B )}$ and $\mathrm{ChAT}+\mathrm{NeuN}+\mathbf{( C )}$ neurons in the CA3 region at 30 days. Data represent the mean $\pm \mathrm{SEM}$. $A N O V A,{ }^{* *} p<0.01(n=5)$. (D) Representative images of ChAT and NeuN positive cells in the CA1 hippocampal subfields of control or 5xFAD mice treated B. bifidum BGN4 and B. longum BORI. Scale bar $=50 \mu \mathrm{m}$. (E,F) Quantifications of the $\mathrm{NeuN}+\mathbf{( E )}$ and $\mathrm{ChAT+} / \mathrm{NeuN}+\mathbf{( F )}$ neurons in the CA1 hippocampal region at 30 days. Data represent the mean \pm SEM. ANOVA, ${ }^{\star} p<0.05,{ }^{* *} p<0.01(n=5)$. (G) Representative images of ChAT and NeuN positive cells in the CA2 hippocampal subfields of control or 5xFAD mice treated B. bifidum BGN4 and B. longum BORI. Scale bar $=50 \mu \mathrm{m}$. (H,I) Quantifications of the $\mathrm{NeuN}+\mathbf{( H )}$ and $\mathrm{ChAT+} / \mathrm{NeuN}+\mathbf{( I )}$ neurons in the $\mathrm{CA} 2$ hippocampal region at 30 days. Data represent the mean $\pm \operatorname{SEM}(n=5)$. 
A

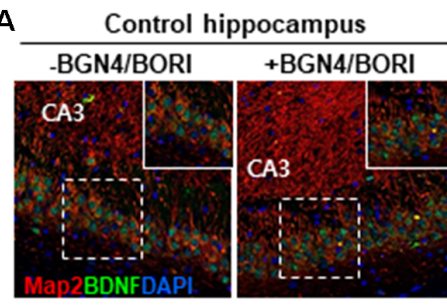

c

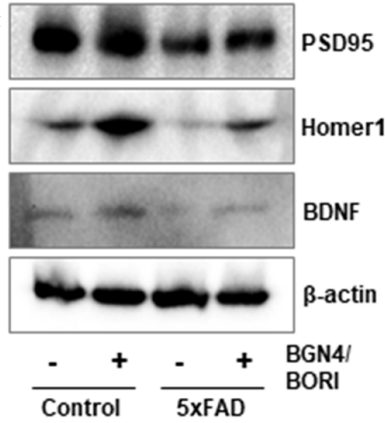

$\mathbf{F}$

Control hippocampus
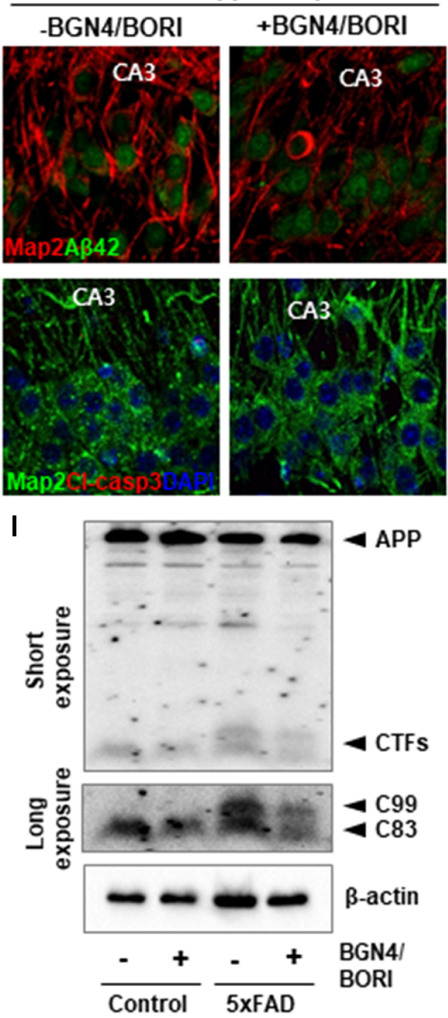
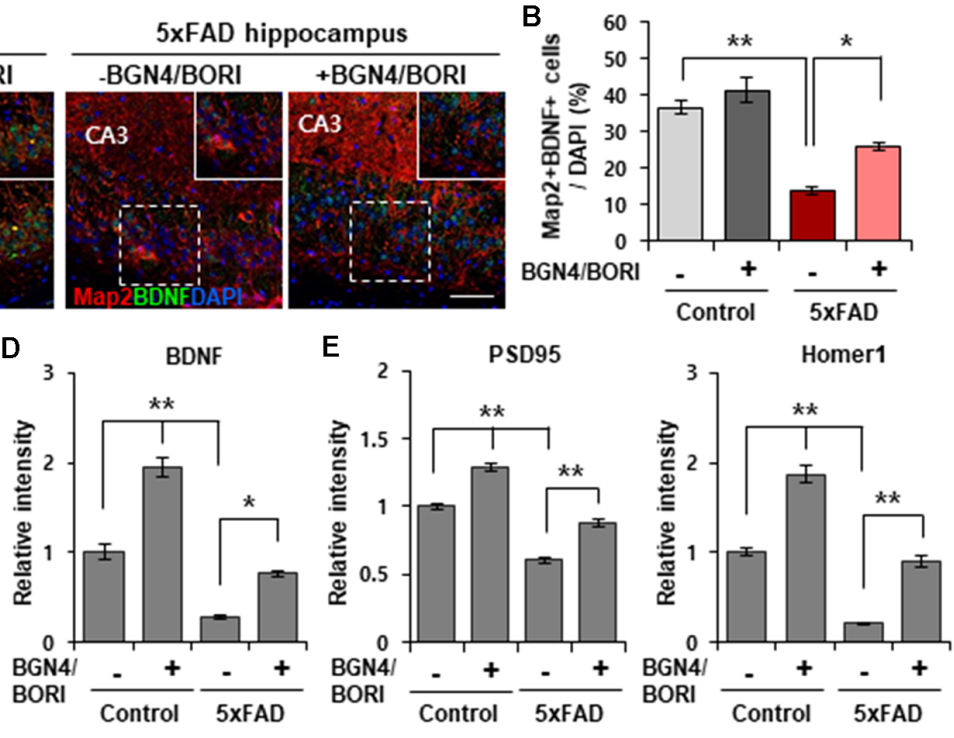

5xFAD hippocampus
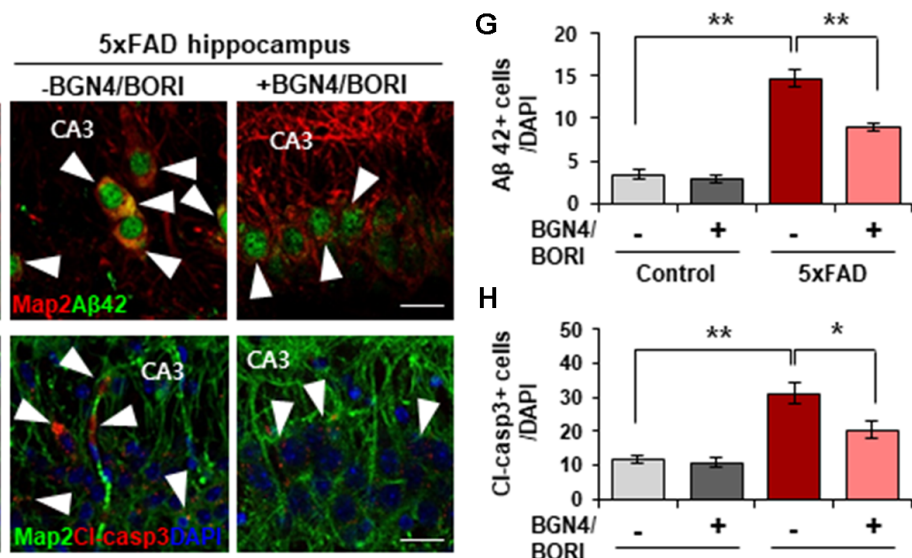

$\mathbf{J}$
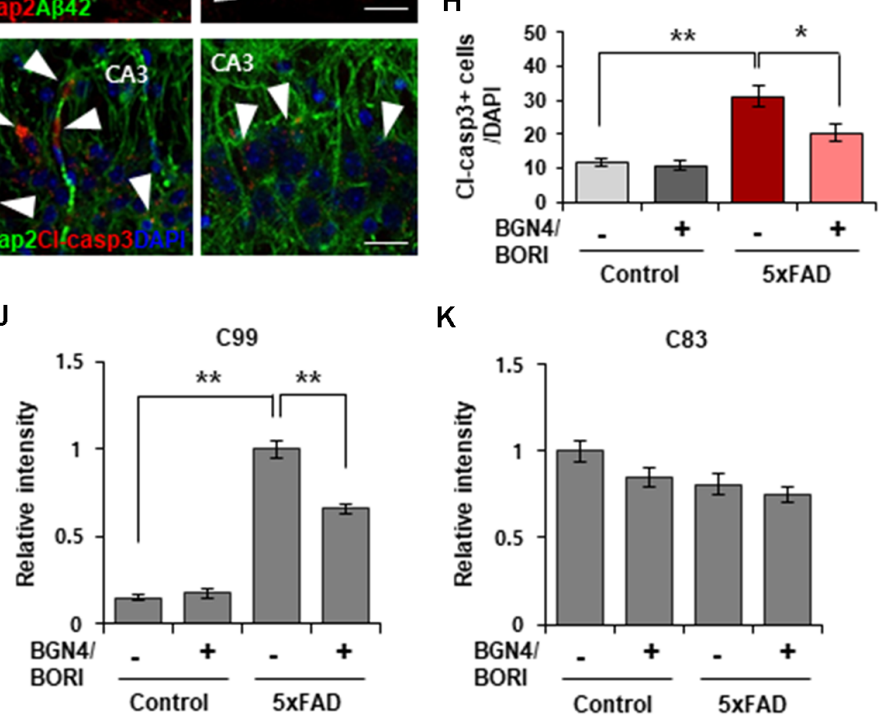

K

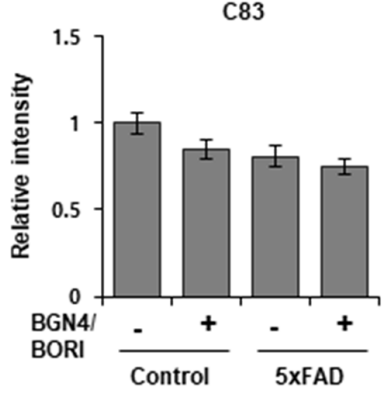

FIGURE 2 | B. bifidum BGN4 and B. longum BORI reduced Alzheimer's disease (AD) pathogenesis in the 5xFAD Mouse Brain. (A) Representative images of Map2 and BDNF positive cells in the CA3 hippocampal subfields of control or 5xFAD mice treated with B. bifidum BGN4 and B. Iongum BORI (BGN4/BORI). Scale bar $=50 \mu \mathrm{m}$. (B) Quantification of the Map2+/BDNF+ neurons in the CA3 hippocampal region at 30 days. Data represent the mean $\pm S E M$. ANOVA, ${ }^{*} P<0.05$, ${ }^{\star *} p<0.01(n=5)$. (C) Western blot analysis of the scaffolding proteins, PSD95 and Homer1, and blood brain-derived neurotrophic factor, BDNF, in the hippocampus of control or 5xFAD mice treated with B. bifidum BGN4 and B. Iongum BORI. (D) Hippocampal quantification of BDNF expression were normalized to $\beta$-actin. Data represent the mean \pm SEM. ANOVA, ${ }^{*} p<0.05,{ }^{* \star} p<0.01(n=5)$. (E) Hippocampal quantifications of PSD95 (left) and Homer1 (right) expression were normalized to $\beta$-actin. Data represent the mean \pm SEM. ANOVA, ${ }^{* *} p<0.01(n=5)$. (F) Representative images showing the production of amyloid- $\beta 42$ (top) and cleaved-caspase3 (bottom) in the CA3 hippocampal subfields of control or 5xFAD mice treated with B. bifidum BGN4 and B. longum BORI. Scale bar $=20 \mu \mathrm{m}$. (G,H) Quantifications of the amyloid- $\beta 42+(\mathbf{G})$ and cleaved-caspase3+ $\mathbf{( H )}$ cells in the CA3 hippocampal region at 30 days. Data represent the mean \pm SEM. ANOVA, ${ }^{*} p<0.05,{ }^{*} p<0.01$ $(n=6)$. (I) Immunodetection of amyloid precursor protein (APP), C99, C83 in Western blots with protein lysates derived from the hippocampus. (J,K) Hippocampal quantifications of C99 (J) and C83 (K) expression were normalized to $\beta$-actin. Data represent the mean \pm SEM. ANOVA, ${ }^{\star \star} p<0.01(n=5)$. 
significantly decreased the expression of $L-17$ and $I L-6$ in $5 x F A D$ mice, whereas no differences in the expression of $I L-1 \beta$ and $I L$ 10 were observed (Figures $\mathbf{3 A}-\mathbf{D}$ ). To determine how the AD phenotype was ameliorated by B. bifidum BGN4 and B. longum BORI, we next assessed the expression of the inflammatory pathway factor, $N F-k B$, and its downstream marker, COX2. Consistently, $N F-k B$ expression was attenuated in B. bifidum BGN4 and B. longum BORI-treated 5xFAD mice, similar to control levels (Figure 3E, right panel). In addition, we confirmed that the expression of COX2 was significantly decreased in probiotic-treated $5 \mathrm{xFAD}$ mice (Figure $3 \mathrm{E}$, left panel). In the serum, the concentration of the proinflammatory proteins, TNF$\alpha$ and IL-12, were significantly restored in $5 x F A D$ mice treated with B. bifidum BGN4 and B. longum BORI (Figure 3F). Finally, we observed that the expression of microglial marker, Iba1, and astrocytic marker, Gfap, were significantly decreased in the hippocampus of probiotic-treated $5 x F A D$ mice compared with control-treated 5xFAD mice (Figures 3G,H). Taken together, these results suggest that $B$. bifidum BGN4 and B. longum BORI ameliorates the neuroinflammatory response, which may prevent the progression of the AD phenotype.

\section{B. bifidum BGN4 and B. longum BORI Attenuated Cognitive Impairments in 5xFAD Mice}

Next, to investigate whether cognitive deficits can be attenuated by B. bifidum BGN4 and B. longum BORI treatment, we assessed spatial recognition memory using spontaneous alternation behavior in the Y-maze test. Remarkably, probiotic-treated $5 \mathrm{xFAD}$ mice showed an improved alternation performance behavior when compared with non-treated 5xFAD mice (Figure 4A). In the contextual fear conditioning test, we found a significant increase in the freezing ratio in $5 \mathrm{xFAD}$ mice treated with B. bifidum BGN4 and B. longum BORI (Figures 4B,C). Next, we sought to determine whether AD-associated memory loss could be rescued by B. bifidum BGN4 and B. longum BORI in the Morris water maze test. Importantly, on probe test without a platform, we found that B. bifidum BGN4 and B. longum BORI treatment dramatically increased time spent in the target quadrant when compared with non-treated 5xFAD mice (Figures 4D,E). These results indicated that $B$. bifidum BGN4 and B. longum BORI treatment improved AD-associated memory deficits in 5xFAD mice.

\section{Altered Gut Microbiome by B. bifidum BGN4 and $B$. longum BORI in 5xFAD Mice}

To assess the effect of probiotic supplementation on intestinal bacterial communities, mouse fecal microbiota profiles were analyzed during supplementation. To collect murine fecal samples, fecal pellets were collected directly from the anal orifices once a week for 5 weeks in the probiotic treated mice. Interestingly, we found that the $5 \mathrm{xFAD}$ mice group fed with B. bifidum BGN4 and B. longum BORI showed depletions in the bacterial genus Parvibacter, Incertae_Sedis, and Oscillibacter, and enrichments in Akkermansia, Faecalibacterium, Erysipelatoclostridium, and Candidatus_Stoquefichus when compared with the control group (LDA >3.0; Figures 5A,B).
Moreover, control mice fed with B. bifidum BGN4 and $B$. longum BORI showed lower relative abundances of the genus NK4A214_group, Alistipes, Lachnoclostridium, Desulfovibrio, and the family Peptococcaceae when compared with non-treated control mice (LDA >3.0; Figures 5A,C). The most relevant change was the enrichment of the genus Akkermansia. Akkermansia was less abundant in the probiotic-treated $5 \mathrm{xFAD}$ mice $(0.25 \%)$ compared with the untreated $5 x F A D$ mice $(1.21 \%)$ before the administration of probiotics; this showed a tendency to increase at weeks $1-3$ in the $5 \mathrm{xFAD}$ mice treated with $B$. bifidum BGN4 and B. longum BORI (1.83-5.86\%) and decrease in the untreated $5 x F A D$ group $(0.15-0.51 \%$; Figure $5 \mathrm{D})$. The relative abundance of Akkermansia was strongly correlated with probiotic supplementation periods $(\rho=-0.81)$. In the $5 x F A D$ mice group treated with B. bifidum BGN4 and B. longum BORI, the marked decline was mitigated, showing a weak negative correlation $(\rho=-0.26$; Figure 5E). Thus, these results indicated that B. bifidum BGN4 and B. longum BORI treatment altered Gut Microbiome in 5xFAD mice.

\section{DISCUSSION}

$\mathrm{AD}$ is the most common dementia. It is caused by an accumulation of $\mathrm{A} \beta$ peptides in the adult brain. Amyloid pathogenesis starts with a nucleation phase that leads to the formation of small aggregates in 2-month-old 5xFAD mice (Oakley et al., 2006). Next, the accumulation of intraneuronal A $\beta$ is rapidly increased during the amyloid growth stage (Takahashi et al., 2002; Eisenberg and Jucker, 2012). Recent studies have identified intestinal microbiome dysbiosis, including decreased Firmicutes, increased Bacteroidetes, and decreased Bifidobacterium, in patients with AD (Vogt et al., 2017; Li et al., 2018). These findings suggested that microbiome modulation via supplementation of a beneficial and safe probiotic can ameliorate physiological functions, such as the epithelial barrier, gut homeostasis, and the inflammatory response, and improve psychiatric and neurological disease symptoms through the regulation of the gut-brain axis (AitBelgnaoui et al., 2014; Akbari et al., 2016). These findings lead us to examine the effects of B. bifidum BGN4 and $B$. longum BORI administration on $\mathrm{AD}$ pathological features, such as amyloid aggregation, neuroinflammatory response, and AD-associated memory.

In our study, we should note that probiotic supplementation was orally administrated to 3 -month-old $5 x F A D$ mice which display early amyloid growth stages; therefore, our data clearly indicate that B. bifidum BGN4 and B. longum BORI treatment can efficiently reduce amyloid aggregation and cellular apoptosis in the early stages of $\mathrm{AD}$. However, since $\mathrm{AD}$ shows a progressive neurodegenerative phenotype, it is important to determine the subsequent effects of this treatment in the middle and late stages of $\mathrm{AD}$. A previous study has demonstrated that treatment with a probiotic mixture containing Lactobacillus acidophilus, Lactobacillus casei, Bifidobacterium bifidum, and Lactobacillus fermentum significantly improves the Mini-mental state examination (MMSE) score of patients with severe AD (Akbari et al., 2016). Further studies are required to 
A

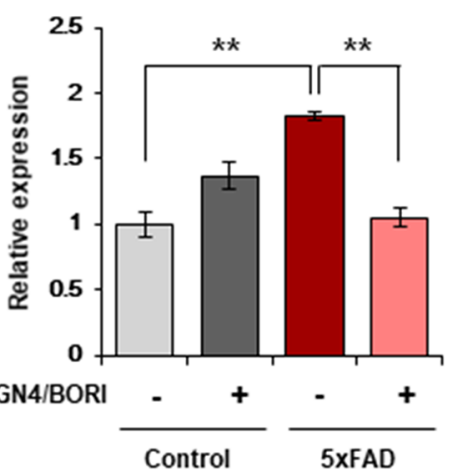

D

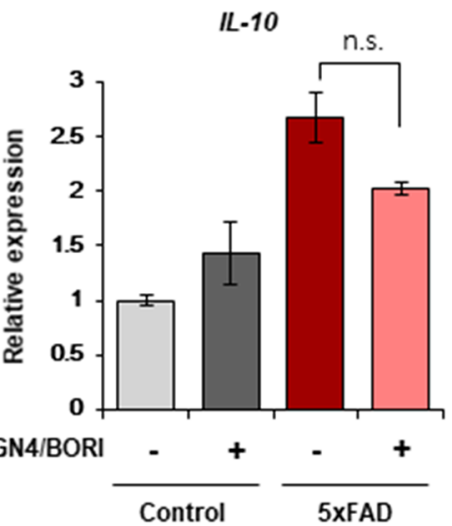

F

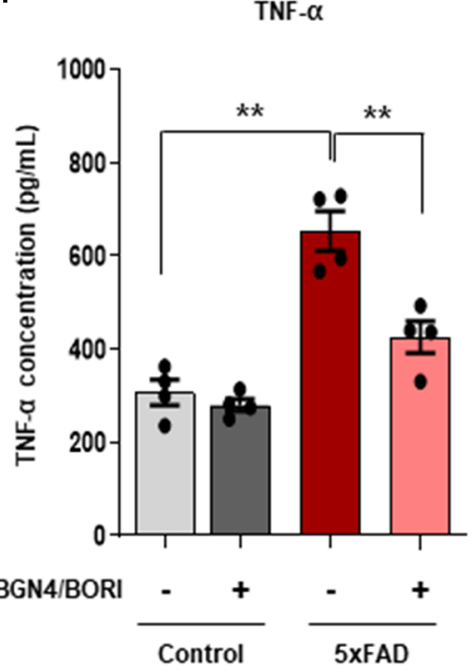

B

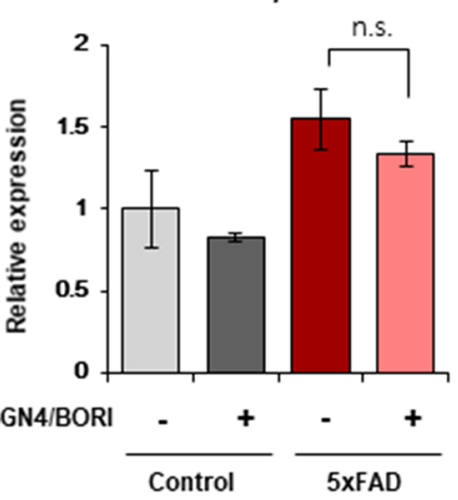

E

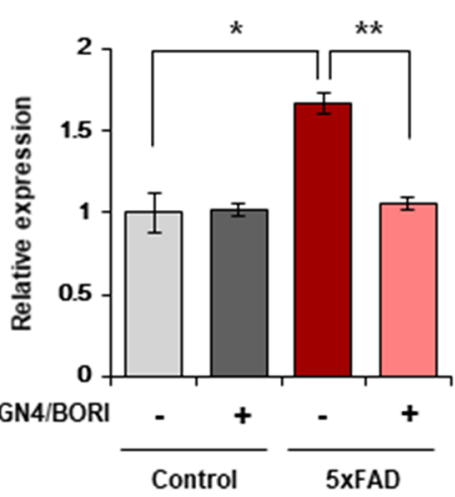

C

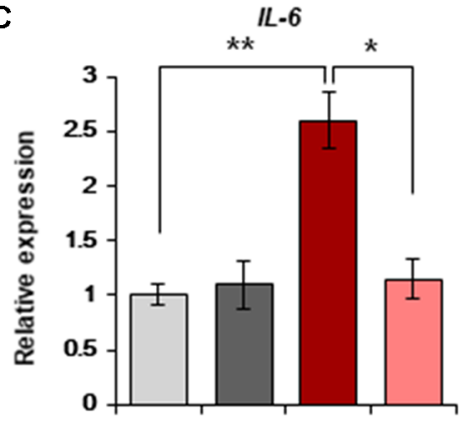

BGN4/BORI

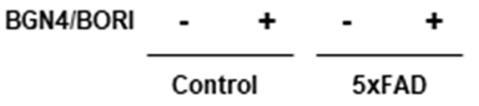

$\operatorname{cox} 2$

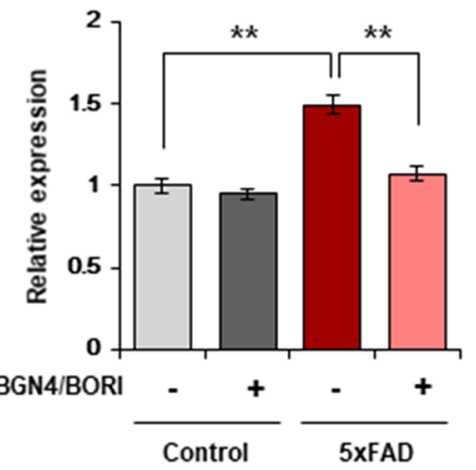

G

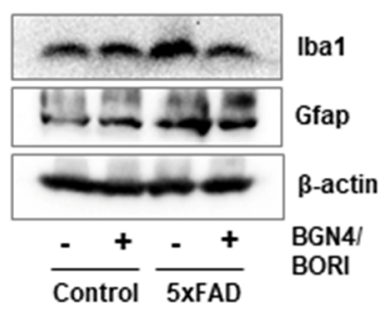

H

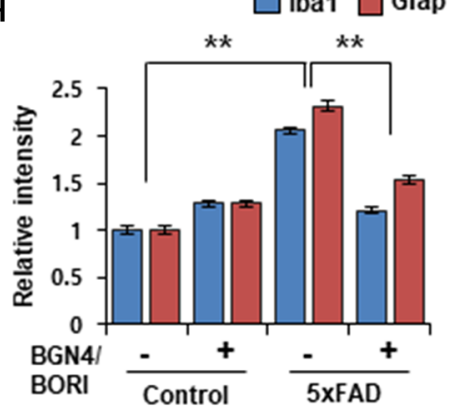

FIGURE 3 | Effects of B. bifidum BGN4 and B. longum BORI on the neuroinflammatory responses in 5xFAD Mice. (A-C) Quantitative RT-PCR analysis of the pro-inflammatory cytokines, $I L-17$ (A), $I L-1 \beta$ (B), and $I L-6$ (C) in the hippocampus of control and 5xFAD mice treated with B. bifidum BGN4 and B. Iongum BORI (BGN4/BORI). Data represent the mean \pm SEM. ANOVA, data represent the mean \pm SEM. ANOVA, ${ }^{*} p<0.05,{ }^{\star *} p<0.01$; n.s., not significant $(n=5)$. (D) Quantitative RT-PCR analysis of the anti-inflammatory cytokine, $I L-10$, in the hippocampus of control or 5xFAD mice treated with B. bifidum BGN4 and B. longum BORI. Data represent the mean \pm SEM, n.s., not significant $(n=5)$. (E) Quantitative RT-PCR analysis of inflammation pathway genes, NF-kB and COX2, in the hippocampus of control or 5XFAD mice treated with B. bifidum BGN4 and B. Iongum BORI. Data represent the mean \pm SEM. ANOVA, ${ }^{*} p<0.05,{ }^{* *} p<0.01(n=5)$. (F) The concentration of TNF- $\alpha$ and IL-12 in the serum of control or 5XFAD mice treated with $B$. bifidum BGN4 and B. longum BORI. Data represent the mean \pm SEM. ANOVA, ${ }^{* *} p<0.01(n=4)$. (G) Immunodetection of lba1 and Gfap in Western blots with protein lysates derived from the hippocampus. (H) Hippocampal quantification of Iba1 and Gfap expression were normalized to $\beta$-actin. Data represent the mean \pm SEM. ANOVA, ${ }^{\star \star} p<0.01(n=5)$. 
A

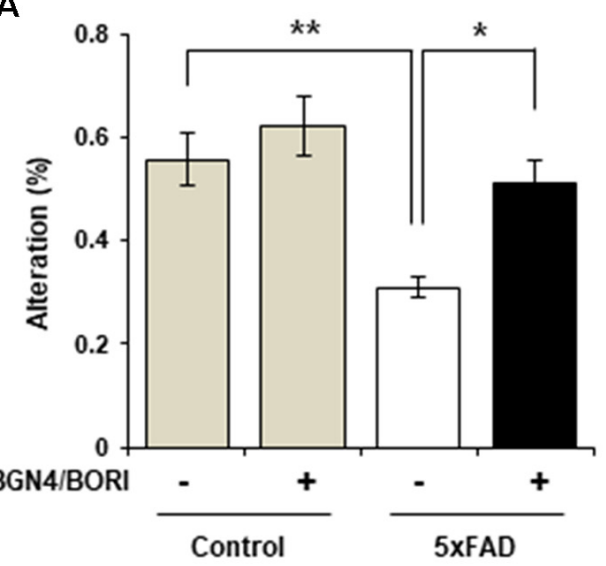

C

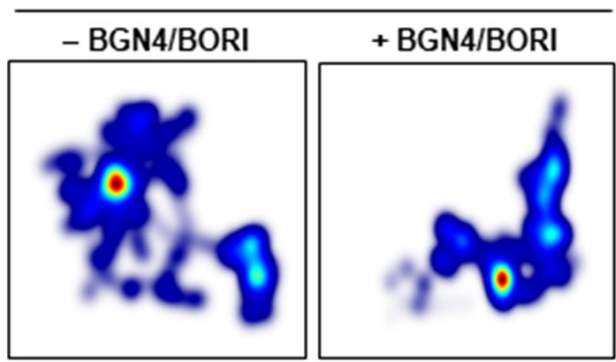

B
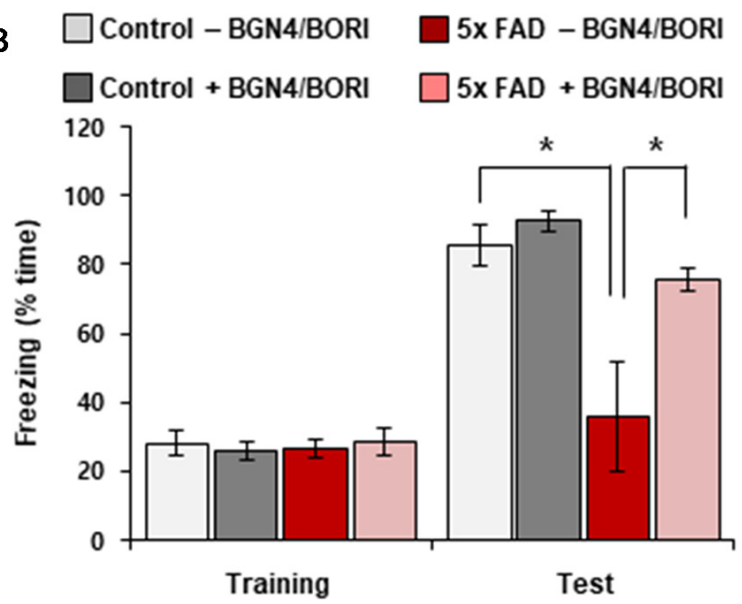

5xFAD mice

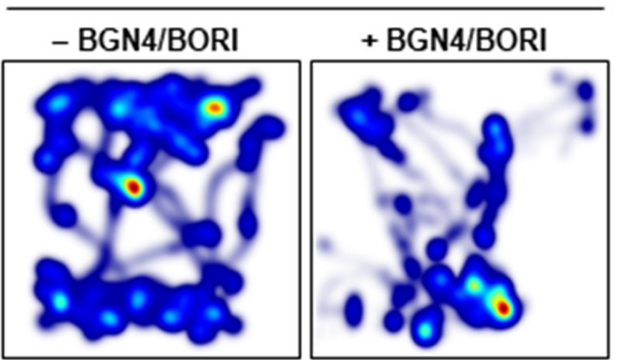

D
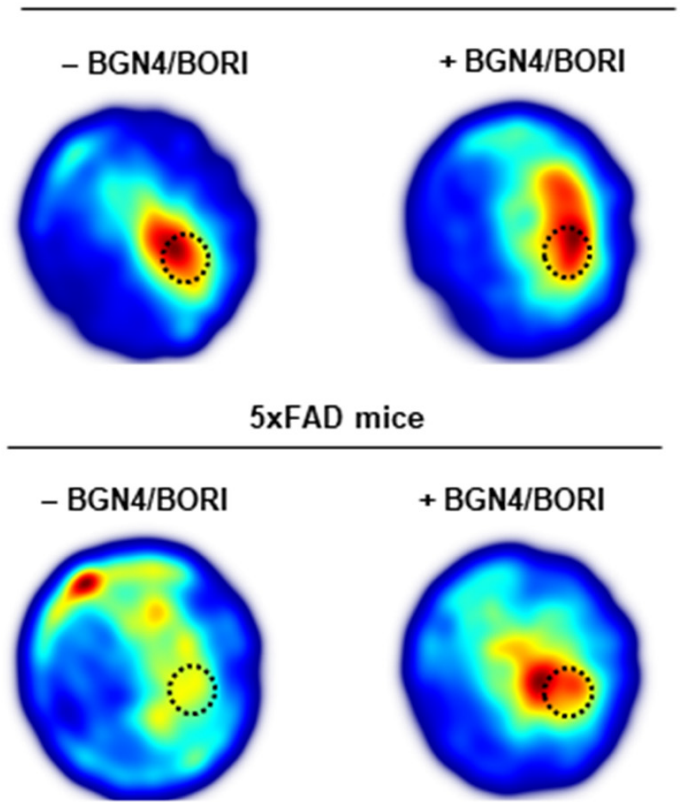

+ BGN4/BORI

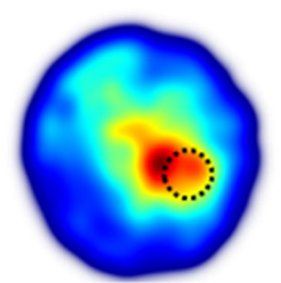

E
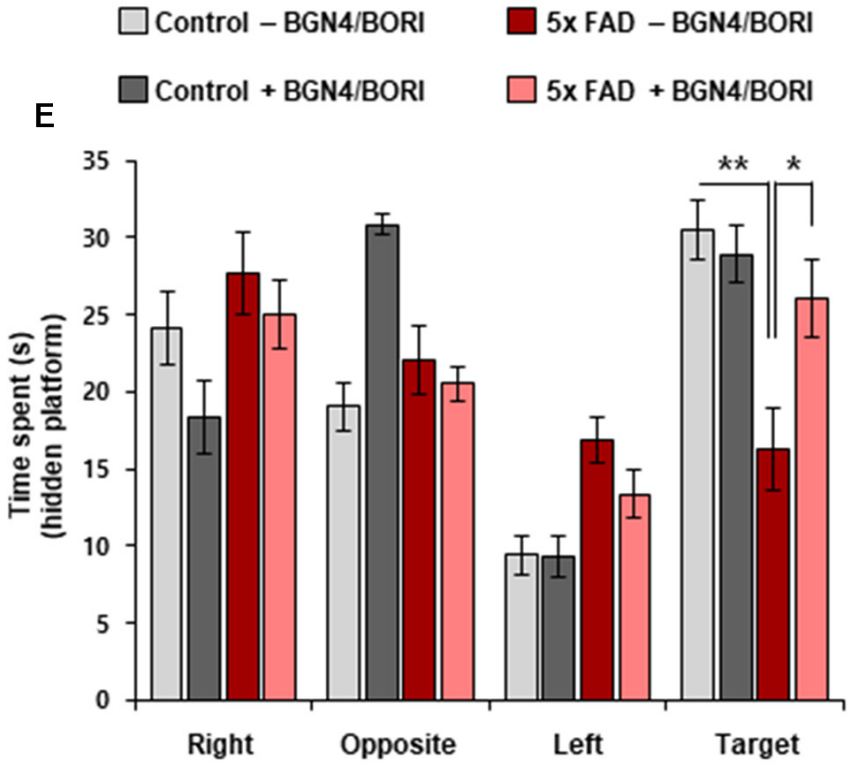

FIGURE 4 | Cognitive impairments was attenuated by B. bifidum BGN4 and B. longum BORI in 5xFAD Mice. (A) Effect of B. bifidum BGN4 and B. longum BORI (BGN4/BORI) treatment on the performance of control and 5xFAD mice in the Y-maze test. The percent spontaneous alternation was recorded over 5-min in each trial. Data represent the mean \pm SEM. ANOVA, ${ }^{*} p<0.05,{ }^{* *} p<0.01$ ( $n=6$ per group). (B) Effect of $B$. bifidum BGN4 and $B$. longum BORI treatment on contextual fear conditioning test. Freezing levels of control $(n=5)$, BGN4/BORI-treated control $(n=5), 5 \times F A D(n=5)$, and BGN4/BORI-treated 5xFAD $(n=6)$ on day 2. Data represent the mean \pm SEM. ANOVA, ${ }^{*} p<0.05$. (C) Representative heatmaps during fear conditioning test. (D) Effect of $B$. bifidum BGN4 and $B$. longum BORI on the performance of control and 5xFAD mice in the Morris water maze. Representative heatmaps showing that the time spent in the circular pool to assess long-term spatial learning memory. The dotted circle indicates the target region. (E) Quantification of duration in the probe quadrant in the prove trial. Data represent the mean \pm SEM. ANOVA, ${ }^{*} p<0.05,{ }^{* *} p<0.01$ ( $n=8$ per each group). 
A

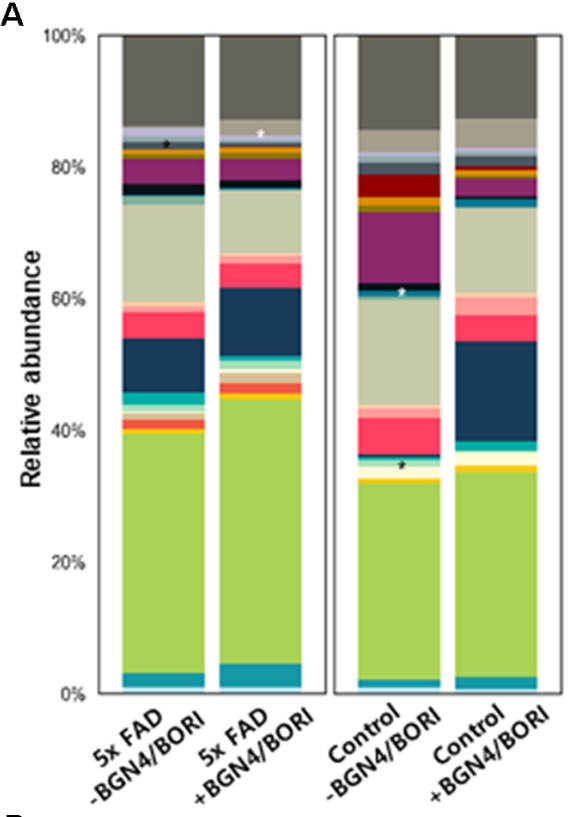

D
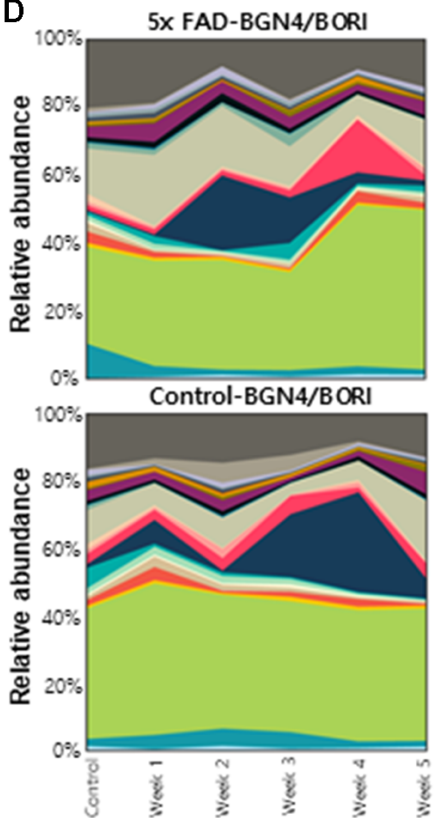

a Minor_taxa

E_Verrucomicroblota;g_Akcermansia

E_Patoscibactoria:g_Candidatus_Saccharimonas

= P_Firmicutos;g_Incortoo_Sedis

n p_Firmicutesf_Oscillospiraceae;

- D_Firmicutos:__RuminococcusL_torques_group

- P_Firmicutes:9_Maminbryantia

- P_Firmicutes:g_Lachnospiraceae_UCG-006

- P_Firmicutos:g_Lachnospiraceee_NK4A136_gro

- P_Firmicutes:9_Lachnoclostridium

E_Firmicutes:g_Blautia

D.Firmicutos:g_A2

D_Firmicutost_Lachnospiracoeo:

IIIF_Firmicutes:g_Candidatus_Arthromitus
B

anoe_tass

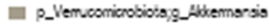

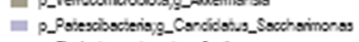

In P-Fniojes,g_lncerise_Secio

- Pfinriostes;_Osclospiescese;

- P_Fniodes;g_[Rumirococoss__brques_group

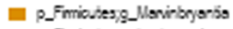

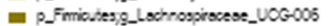

- P_Finiostes;__Lochrospirseses_NKAA136_goup

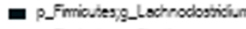

- P_Finiodes;g_Blosio

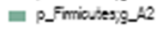

P_Fmiostes;_Loshnospiosese;-

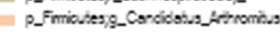

En P_Fmiodes;_Closticio_UOG-014

E P_Finiodes;g_Loctobscil.s

a P_Finiodes;s_Oubosiclo

- P_Complobsciteriota;g_Hdicobsciter

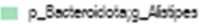

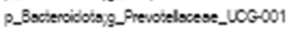

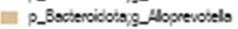

- P_Bsciencicota,_-Prevotalscese;-

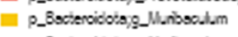

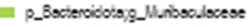

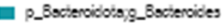

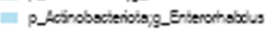

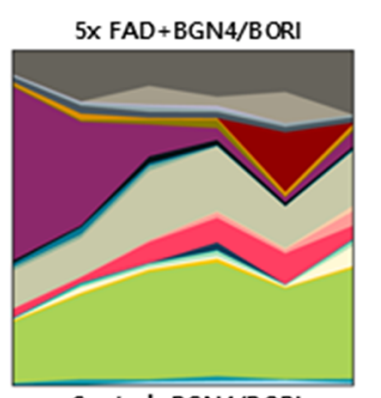

E

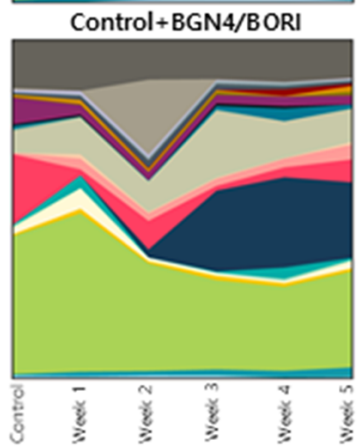

= D_Firmicutos:g_Clostricia_UCG-014 n_Firmicutes:g_Lactobecillus

- P_Firmicutes:g_Dubosiella

- p_Campliobacterota;__Helicobacter

= P_Bacteroidota:9_Alistipes

P_Bacteroidota:9_Prevotellaceae_UCG-001

In_E_Bctordidota:g_Alloprovotolla

- p_Bectoroidota;_Provotellacoeo:

In_Bacteroidota;9_Murbaculum

= P_Bscteroldota;g_Murbaculaceae

- P_Bactercidota;g_Bacteroides

In_Actinobacteriota:g_Enterorhabdus

C
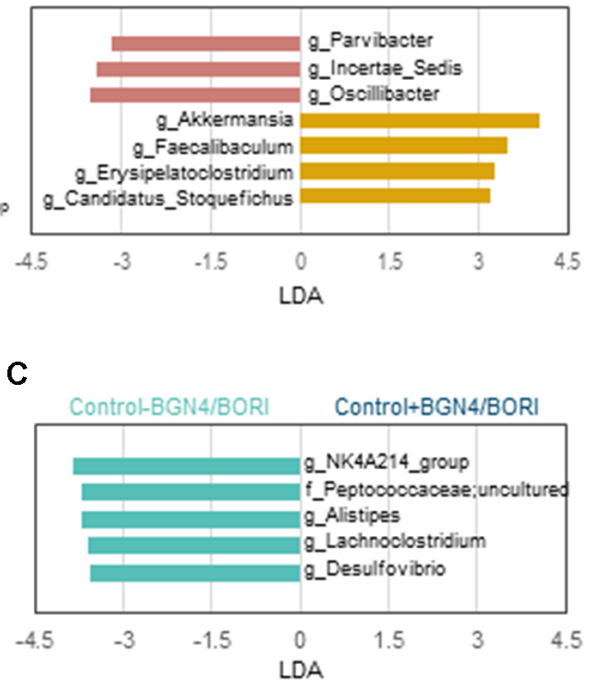

C

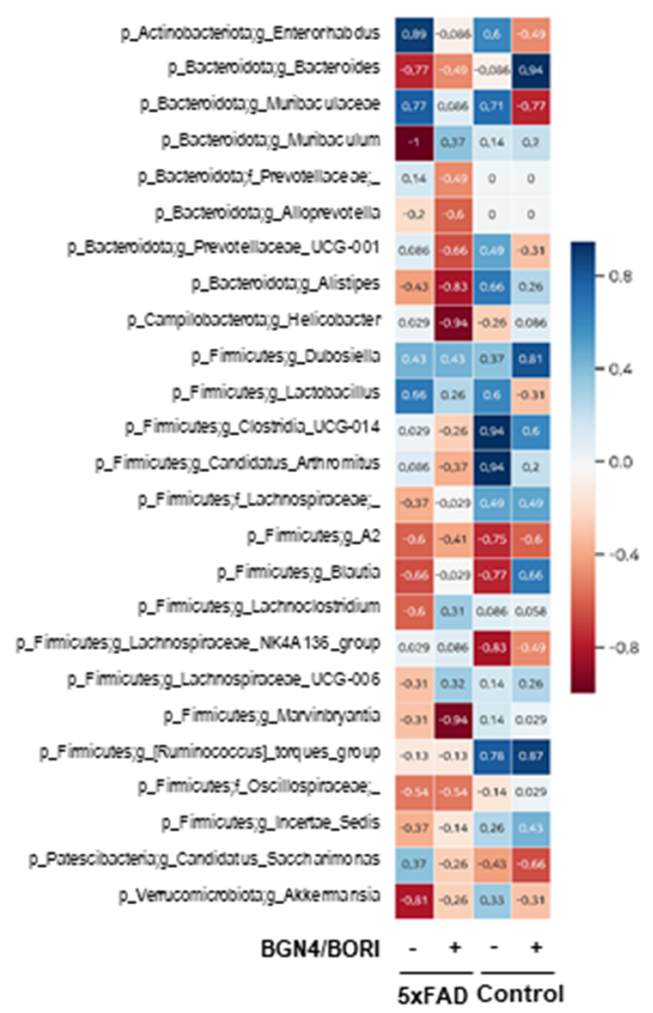

5xFAD Control

FIGURE 5 | Differences in the gut microbiome following B. bifidum BGN4 and B. longum BORI Treatment in 5xFAD Mice. (A) Comparison of differences in microbial composition between the probiotics intake and the non-intake group (Week 1-5 samples) of 5xFAD or control mice. *Indicates linear discriminant analysis (LDA) score >3.0. Microbial taxa differing (B) between 5xFAD and BGN4/BORI-treated 5xFAD mice and (C) between control and BGN4/BORI-treated control mice determined by LEfSe analysis (LDA scores >3.0 are shown). (D) Changes in microbial composition at genus level in each group over time. (E) Spearman's rank correlation showing the relationship between bacterial abundance at the genus level and time. 
assess whether probiotic supplementation attenuates the progression of the $\mathrm{AD}$ phenotype during the mid and late amyloid-seeding stages.

Moreover, we found that $5 x F A D$ mice group treated with B. bifidum BGN4 and B. longum BORI, depleted bacterial from the genus, Parvibacter, Incertae_Sedis, and Oscillibacter, and enriched Bifidobacterium, Akkermansia, Faecalibacterium, Erysipelatoclostridium, and Candidatus_Stoquefichus, when compared with untreated $5 x F A D$ mice. Among them, the most relevant change was the enrichment of the genus Akkermansia and Faecalibacterium. Both bacteria possess anti-inflammatory properties (Thursby and Juge, 2017). Interestingly, supplementation of Akkermansia muciniphila reduces $\mathrm{A} \beta$ 40-42 in the cerebral cortex of APP/PS1 mice, shortens study time, and improves completion rate in Y-maze tests (Ou et al., 2020). Further, this treatment improves intestinal barrier function. Another interesting genus that was enriched was Faecalibacterium. This is a representative butyrate-producing bacteria; this substance acts as an anti-inflammatory agent by suppressing the nuclear factor kappa-light-chain-enhancer of the signaling pathways of activated B cells (Schwab et al., 2007). Butyrate drives microglial maturation and is required for the maintenance of mature microglia (Cresci and Bawden, 2015). However, fecal microbiota may not be fully representative of those in the contents or mucosa of the gastrointestinal (GI) tract (Lyra et al., 2012; Lavelle et al., 2015). Thus, the expression of microbial population in fecal samples might be quite different to the intestinal population, which has the capacity to induce changes in the brain. Nevertheless, because of the convenience and non-invasiveness of fecal sampling, many studies have used fecal samples as a proxy to study the gut microbiota. Therefore, a comprehensive understanding of microbial populations between fecal and GI microbiota would help improve longitudinal analyses of microbiota and the application of fecal samples (Lo Presti et al., 2019).

We also explored the neuroinflammatory response that contributes to the progression of $\mathrm{AD}$ pathology. Our results showed that probiotic treatment mitigated neuronal inflammation and elevated BDNF expression in AD mice. Previous studies have demonstrated that activated microglia cells were accompanied by increased pro-inflammatory cytokines, such as interleukins and TNF- $\alpha$, in the AD brain (Vukic et al., 2009; Wang et al., 2015). However, these activated microglia switch to the anti-inflammatory M1-like phenotype which secrete anti-inflammatory cytokines, such as interleukin-4 and interleukin-10, and BDNF that are responsible for inhibiting the innate and adaptive immune reaction and restore synaptic function (Sánchez-Sarasúa et al., 2020). Consistent with this, elevating BDNF expression in the hippocampus of $5 \mathrm{xFAD}$ mice was associated with improved cognition (Choi et al., 2018). Thus, our results support that treatment of the B. bifidum BGN4 and B. longum BORI effectively improved the cognitive functions through an increased BDNF and a decreased neuroinflammatory response in the $\mathrm{AD}$ mice.
Moreover, we showed the increase in the synaptic plasticity by oral administration of the B. bifidum BGN4 and B. longum BORI in control mice (Figures 2C-E), suggesting that altered gut microbiota can modulate the synaptic function through the gut-brain axis even in the control healthy mice. Since increased synaptic plasticity by oral administration of the B. bifidum BGN4 and B. longum BORI was not previously reported in control mice, our findings provide important implications for the neurotherapeutic effect of the B. bifidum BGN4 and B. longum BORI.

Taken together, our results demonstrated that treatment with B. bifidum BGN4 and B. longum BORI ameliorated cognitive dysfunction and memory loss in an $\mathrm{AD}$ mouse model via elevated BDNF expression. These results indicate the therapeutic potential of B. bifidum BGN4 and B. longum BORI in preventing the pathological features of $\mathrm{AD}$.

\section{CONCLUSION}

Our data provide evidence for a therapeutic potential of $B$. bifidum BGN4 and B. longum BORI in the mouse model of AD. Administration of B. bifidum BGN4 and B. longum BORI effectively improves cognition and memory through an increased BDNF and a decreased neuroinflammatory response in the mouse AD hippocampus. Thus, these results indicated that oral treatment with B. bifidum BGN4 and B. longum BORI could be a novel therapeutic for $\mathrm{AD}$. In the future, it is necessary to verify the efficacy of B. bifidum BGN4 and B. longum BORI in humans for clinical applications.

\section{DATA AVAILABILITY STATEMENT}

Publicly available datasets were analyzed in this study. This data can be found here: NCBI BioProject, https://www.ncbi.nlm.nih.gov/Traces/study/?acc=PRJNA731317.

\section{ETHICS STATEMENT}

The animal study was reviewed and approved by Institutional Animal Care and Use Committee at Dongguk University (IACUC-2021-006-1).

\section{AUTHOR CONTRIBUTIONS}

HK and SK performed the experiments. HK, S-jP, GP, HS, and MP performed the data analysis. $\mathrm{HK}, \mathrm{MP}$, and JK designed the study and contributed to writing the manuscript. All authors contributed to the article and approved the submitted version.

\section{FUNDING}

This research was supported by the Ministry of Small and Medium-sized Enterprises (SMEs) and Startup (MSS), Korea, under the "Regional Specialized Industry Development Plus Program (R\&D, S2848321)" supervised by the Korea Institute for Advancement of Technology (KIAT). 


\section{REFERENCES}

Ait-Belgnaoui, A., Colom, A., Braniste, V., Ramalho, L., Marrot, A., Cartier, C., et al. (2014). Probiotic gut effect prevents the chronic psychological stressinduced brain activity abnormality in mice. Neurogastroenterol. Motil. 26, 510-520. doi: 10.1111/nmo.12295

Akbari, E., Asemi, Z., Daneshvar Kakhaki, R., Bahmani, F., Kouchaki, E., Tamtaji, O. R., et al. (2016). Effect of probiotic supplementation on cognitive function and metabolic status in Alzheimer's disease: a randomized, doubleblind and controlled trial. Front. Aging Neurosci. 8:256. doi: 10.3389/fnagi.2016. 00256

Bokulich, N. A., Kaehler, B. D., Rideout, J. R., Dillon, M., Bolyen, E., Knight, R., et al. (2018). Optimizing taxonomic classification of marker-gene amplicon sequences with QIIME 2's q2-feature-classifier plugin. Microbiome 6:90. doi: 10.1186/s40168-018-0470-z

Cattaneo, A., Cattane, N., Galluzzi, S., Provasi, S., Lopizzo, N., Festari, C., et al. (2017). Association of brain amyloidosis with pro-inflammatory gut bacterial taxa and peripheral inflammation markers in cognitively impaired elderly. Neurobiol. Aging 49, 60-68. doi: 10.1016/j.neurobiolaging.2016. 08.019

Checler, F. (1995). Processing of the beta-amyloid precursor protein and its regulation in Alzheimer's disease. J. Neurochem. 65, 1431-1444. doi: 10.1046/j. 1471-4159.1995.65041431.x

Chen, L., Na, R., Boldt, E., and Ran, Q. (2015). NLRP3 inflammasome activation by mitochondrial reactive oxygen species plays a key role in long-term cognitive impairment induced by paraquat exposure. Neurobiol. Aging 36, 2533-2543. doi: 10.1016/j.neurobiolaging.2015.05.018

Choi, S. H., Bylykbashi, E., Chatila, Z. K., Lee, S. W., Pulli, B., Clemenson, G. D., et al. (2018). Combined adult neurogenesis and BDNF mimic exercise effects on cognition in an Alzheimer's mouse model. Science 361:eaan8821. doi: $10.1126 /$ science.aan 8821

Conley, M. N., Wong, C. P., Duyck, K. M., Hord, N., Ho, E., Sharpton, T. J., et al. (2016). Aging and serum MCP-1 are associated with gut microbiome composition in a murine model. PeerJ 4:e1854. doi: 10.7717/peerj.1854

Cresci, G. A., and Bawden, E. (2015). Gut microbiome: what we do and don't know. Nutr. Clin. Pract. 30, 734-746. doi: 10.1177/0884533615609899

Eisenberg, D., and Jucker, M. (2012). The amyloid state of proteins in human diseases. Cell 148, 1188-1203. doi: 10.1016/j.cell.2012.02.022

El-Sayyad, H. I. (2015). Cholesterol overload impairing cerebellar function: the promise of natural products. Nutrition 31, 621-630. doi: 10.1016/j.nut.2014. 10.017

Goate, A., Chartier-Harlin, M. C., Mullan, M., Brown, J., Crawford, F., Fidani, L., et al. (1991). Segregation of a missense mutation in the amyloid precursor protein gene with familial Alzheimer's disease. Nature 349, 704-706. doi: 10.1038/349704a0

Grenham, S., Clarke, G., Cryan, J., and Dinan, T. (2011). Brain-gut-microbe communication in health and disease. Front. Physiol. 2:94. doi: 10.3389/fphys. 2011.00094

Han, D., Li, Z., Liu, T., Yang, N., Li, Y., He, J., et al. (2020). Prebiotics regulation of intestinal microbiota attenuates cognitive dysfunction induced by surgery stimulation in APP/PS1 mice. Aging Dis. 11, 1029-1045. doi: 10.14336/AD. 2020.0106

Hawley, W. R., Witty, C. F., Daniel, J. M., and Dohanich, G. P. (2015). Choline acetyltransferase in the hippocampus is associated with learning strategy preference in adult male rats. Behav. Brain Res. 289, 118-124. doi: 10.1016/j. bbr.2015.04.034

Hu, N., Yu, J. T., Tan, L., Wang, Y.L., Sun, L., Tan, L., et al. (2013). Nutrition and the risk of Alzheimer's disease. Biomed. Res. Int. 2013:524820. doi: 10.1155/2013/524820

Innes, K. E., Selfe, T. K., Brundage, K., Montgomery, C., Wen, S., Kandati, S., et al. (2018). Effects of meditation and music-listening on blood biomarkers of cellular aging and Alzheimer's disease in adults with subjective cognitive decline: an exploratory randomized clinical trial. J. Alzheimers Dis. 66, 947-970. doi: 10.3233/JAD-180164

Jia, Y., Zhang, X., Yu, J., Han, J., Yu, T., Shi, J., et al. (2017). Acupuncture for patients with mild to moderate Alzheimer's disease: a randomized controlled trial. BMC Complement. Altern. Med. 17:556. doi: 10.1186/s12906-017-2064-x
Jucker, M., and Walker, L. C. (2011). Pathogenic protein seeding in Alzheimer disease and other neurodegenerative disorders. Ann. Neurol. 70, 532-540. doi: 10.1002/ana.22615

Kauwe, J. S., Bailey, M. H., Ridge, P. G., Perry, R., Wadsworth, M. E. Hoyt, K. L., et al. (2014). Genome-wide association study of CSF levels of 59 alzheimer's disease candidate proteins: significant associations with proteins involved in amyloid processing and inflammation. PLoS Genet. 10:e1004758. doi: 10.1371/journal.pgen.1004758

Kim, C. S., Cha, L., Sim, M., Jung, S., Chun, W. Y., Baik, H. W., et al. (2021). Probiotic supplementation improves cognitive function and mood with changes in gut microbiota in community-dwelling older adults: a randomized, double-blind, placebo-controlled, multicenter trial. J. Gerontol. A Biol. Sci. Med. Sci. 76, 32-40. doi: 10.1093/gerona/glaa090

Kim, S., Kwon, S. H., Kam, T. I., Panicker, N., Karuppagounder, S. S., Lee, S., et al. (2019). Transneuronal propagation of pathologic $\alpha$-synuclein from the gut to the brain models parkinson's disease. Neuron 103, 627-641.e7. doi: 10.1016/j.neuron.2019.05.035

Kobayashi, Y., Sugahara, H., Shimada, K., Mitsuyama, E., Kuhara, T., Yasuoka, A., et al. (2017). Therapeutic potential of Bifidobacterium breve strain A1 for preventing cognitive impairment in Alzheimer's disease. Sci. Rep. 7:13510. doi: 10.1038/s41598-017-13368-2

Lauritzen, I., Pardossi-Piquard, R., Bauer, C., Brigham, E., Abraham, J. D., Ranaldi, S., et al. (2012). The $\beta$-secretase-derived C-terminal fragment of $\beta A P P, C 99$, but not $A \beta$, is a key contributor to early intraneuronal lesions in triple-transgenic mouse hippocampus. J. Neurosci. 32, a16243-a16255. doi: 10.1523/JNEUROSCI.2775-12.2012

Lavelle, A., Lennon, G., O'sullivan, O., Docherty, N., Balfe, A., Maguire, A., et al. (2015). Spatial variation of the colonic microbiota in patients with ulcerative colitis and control volunteers. Gut 64, 1553-1561. doi: 10.1136/gutjnl-2014307873

Lee, J. Y., Tuazon, J. P., Ehrhart, J., Sanberg, P. R., and Borlongan, C. V. (2019). Gutting the brain of inflammation: a key role of gut microbiome in human umbilical cord blood plasma therapy in Parkinson's disease model. J. Cell. Mol. Med. 23, 5466-5474. doi: 10.1111/jcmm.14429

Li, Z., Zhu, H., Zhang, L., and Qin, C. (2018). The intestinal microbiome and Alzheimer's disease: a review. Animal Model. Exp. Med. 1, 180-188. doi: 10.1002/ame2.12033

Lo Presti, A., Zorzi, F., Del Chierico, F., Altomare, A., Cocca, S., Avola, A., et al. (2019). Fecal and mucosal microbiota profiling in irritable bowel syndrome and inflammatory bowel disease. Front. Microbiol. 10:1655. doi: 10.3389/fmicb. 2019.01655

Lozupone, C., and Knight, R. (2005). UniFrac: a new phylogenetic method for comparing microbial communities. Appl. Environ. Microbiol. 71, 8228-8235. doi: 10.1128/AEM.71.12.8228-8235.2005

Lozupone, C. A., Hamady, M., Kelley, S. T., and Knight, R. (2007). Quantitative and qualitative beta diversity measures lead to different insights into factors that structure microbial communities. Appl. Environ. Microbiol. 73, 1576-1585. doi: 10.1128/AEM.01996-06

Lyra, A., Forssten, S., Rolny, P., Wettergren, Y., Lahtinen, S. J., Salli, K., et al. (2012). Comparison of bacterial quantities in left and right colon biopsies and faeces. World J. Gastroenterol. 18, 4404-4411. doi: 10.3748/wjg.v18.i32.4404

Matt, S. M., Allen, J. M., Lawson, M. A., Mailing, L. J., Woods, J. A., Johnson, R. W., et al. (2018). Butyrate and dietary soluble fiber improve neuroinflammation associated with aging in mice. Front. Immunol. 9:1832. doi: 10.3389/fimmu. 2018.01832

Mendiola-Precoma, J., Berumen, L. C., Padilla, K., and Garcia-Alcocer, G. (2016) Therapies for prevention and treatment of Alzheimer's disease. Biomed. Res. Int. 2016:2589276. doi: 10.1155/2016/2589276

Oakley, H., Cole, S. L., Logan, S., Maus, E., Shao, P., Craft, J., et al. (2006). Intraneuronal beta-amyloid aggregates, neurodegeneration and neuron loss in transgenic mice with five familial Alzheimer's disease mutations: potential factors in amyloid plaque formation. J. Neurosci. 26, 10129-10140. doi: 10.1523/JNEUROSCI.1202-06.2006

Ou, Z., Deng, L., Lu, Z., Wu, F., Liu, W., Huang, D., et al. (2020). Protective effects of Akkermansia muciniphila on cognitive deficits and amyloid pathology in a mouse model of Alzheimer's disease. Nutr. Diabetes 10:12. doi: 10.1038/s41387020-0115-8 
Price, M. N., Dehal, P.S., and Arkin, A.P. (2010). FastTree 2--approximately maximum-likelihood trees for large alignments. PLoS One 5:e9490. doi: 10.1371/journal.pone.0009490

Sánchez-Sarasúa, S., Fernández-Pérez, I., Espinosa-Fernández, V., SánchezPérez, A. M., and Ledesma, J. C. (2020). Can we treat neuroinflammation in Alzheimer's disease? Int. J. Mol. Sci. 21:8751. doi: 10.3390/ijms21228751

Schwab, M., Reynders, V., Loitsch, S., Steinhilber, D., Stein, J., Schröder, O., et al. (2007). Involvement of different nuclear hormone receptors in butyrate-mediated inhibition of inducible NF kappa B signalling. Mol. Immunol. 44, 3625-3632. doi: 10.1016/j.molimm.2007. 04.010

Segata, N., Izard, J., Waldron, L., Gevers, D., Miropolsky, L., Garrett, W. S., et al. (2011). Metagenomic biomarker discovery and explanation. Genome. Biol. 12:R60. doi: 10.1186/gb-2011-12-6-r60

Sgritta, M., Dooling, S. W., Buffington, S. A., Momin, E. N., Francis, M. B., Britton, R. A., et al. (2019). Mechanisms underlying microbial-mediated changes in social behavior in mouse models of autism spectrum disorder. Neuron 101, 246-259.e246. doi: 10.1016/j.neuron.2018.11.018

Takahashi, R. H., Milner, T. A., Li, F., Nam, E. E., Edgar, M. A., Yamaguchi, H., et al. (2002). Intraneuronal Alzheimer abeta42 accumulates in multivesicular bodies and is associated with synaptic pathology. Am. J. Pathol. 161, 1869-1879. doi: 10.1016/s0002-9440(10)64463-x

Thaipisuttikul, P., and Galvin, J. E. (2012). Use of medical foods and nutritional approaches in the treatment of Alzheimer's disease. Clin. Pract. (Lond) 9, 199-209. doi: 10.2217/cpr.12.3

Thursby, E., and Juge, N. (2017). Introduction to the human gut microbiota. Biochem. J. 474, 1823-1836. doi: 10.1042/BCJ20160510

Vaynman, S., Ying, Z., and Gomez-Pinilla, F. (2004). Hippocampal BDNF mediates the efficacy of exercise on synaptic plasticity and cognition. Eur J. Neurosci. 20, 2580-2590. doi: 10.1111/j.1460-9568.2004.03720.x

Vogt, N. M., Kerby, R. L., Dill-Mcfarland, K. A., Harding, S. J., Merluzzi, A. P., Johnson, S. C., et al. (2017). Gut microbiome alterations in Alzheimer's disease. Sci. Rep. 7:13537. doi: 10.1038/s41598-017-13601-y

Vukic, V., Callaghan, D., Walker, D., Lue, L. F., Liu, Q. Y., Couraud, P. O., et al. (2009). Expression of inflammatory genes induced by beta-amyloid peptides in human brain endothelial cells and in Alzheimer's brain is mediated by the JNK-AP1 signaling pathway. Neurobiol. Dis. 34, 95-106. doi: 10.1016/j.nbd. 2008.12.007

Wang, Y., Cella, M., Mallinson, K., Ulrich, J. D., Young, K. L., Robinette, M. L., et al. (2015). TREM2 lipid sensing sustains the microglial response in an Alzheimer's disease model. Cell 160, 1061-1071. doi: 10.1016/j.cell.2015. 01.049

Wolfe, M. S., Xia, W., Ostaszewski, B. L., Diehl, T. S., Kimberly, W. T., Selkoe, D. J., et al. (1999). Two transmembrane aspartates in presenilin-1 required for presenilin endoproteolysis and gamma-secretase activity. Nature 398, 513-517. doi: $10.1038 / 19077$

Zhan, X., Stamova, B., Jin, L. W., Decarli, C., Phinney, B., and Sharp, F.R. (2016). Gram-negative bacterial molecules associate with Alzheimer disease pathology. Neurology 87, 2324-2332. doi: 10.1212/WNL.0000000000003391

Conflict of Interest: S-jP and MP are directly employed by Bifido Co., Ltd., and hold BIFIDO Co., Ltd. Stocks as a CTO.

The remaining authors declare that the research was conducted in the absence of any commercial or financial relationships that could be construed as a potential conflict of interest.

Publisher's Note: All claims expressed in this article are solely those of the authors and do not necessarily represent those of their affiliated organizations, or those of the publisher, the editors and the reviewers. Any product that may be evaluated in this article, or claim that may be made by its manufacturer, is not guaranteed or endorsed by the publisher.

Copyright (c) 2021 Kim, Kim, Park, Park, Shin, Park and Kim. This is an open-access article distributed under the terms of the Creative Commons Attribution License (CC BY). The use, distribution or reproduction in other forums is permitted, provided the original author(s) and the copyright owner(s) are credited and that the original publication in this journal is cited, in accordance with accepted academic practice. No use, distribution or reproduction is permitted which does not comply with these terms. 